'Facultad de Ciencias de la Salud y de los Alimentos. Universidad del Bío-Bío. Chillán, Chile. 2Escuela de Nutrición y Dietética, Facultad de Medicina-Clínica Alemana, Universidad del Desarrollo.Santiago, Chile.

${ }^{3} F a c u l t a d$ de Ciencias Médicas, Universidad de Santiago. Santiago, Chile. aphD Nutricionista. ${ }^{b} \mathrm{Mg}$, Nutricionista. 'Magíster en Ciencias de la Salud con mención Nutrición.

Este trabajo no recibió financiamiento.

Los autores declaran no tener conflictos de interés.

Recibido el 4 de agosto de 2020, aceptado el 13 de abril de 2021 .

Correspondencia a: Anna Christina Pinheiro Fermandes.

Dirección: Av Plaza, 680, Las Condes, Santiago. apinheiro@udd.cl

\section{Inseguridad alimentaria y hábitos alimentarios durante la pandemia de COVID-19 en hogares chilenos}

\author{
JACQUELINE ARANEDA ${ }^{1, \mathrm{a}}$, ANNA CHRISTINA PINHEIRO ${ }^{2, \mathrm{~b}}$, \\ TITO PIZARRO ${ }^{3, \mathrm{c}}$
}

\section{Effects of COVID-19 pandemic on food insecurity perception in Chilean households}

Background: The coronavirus 2 pandemic (COVID-19) has caused intense changes in lifestyles, feeding, and nutrition habits. Aim: To analyze food insecurity and eating habits during the period of quarantine in a group of Chilean individuals. Material and Methods: An online survey was conducted in 2,767 households with questions about the sociodemographic composition of the household, household food purchasing habits in the quarantine period for COVID-19, and perception of food insecurity. Results: More than $60 \%$ of households $(p<0.01)$ were worried about not having enough food, especially when children under 18 years of age lived in them. This perception increased in urban households $(p<0.01)$ and those located in the Central-Southern and Southern regions $(p<0.01)$. During the pandemic, the consumption of healthy products such as milk increased in $50 \%$ of households, legumes in $72 \%$, eggs in $79 \%$, fruits in $54 \%$, vegetables in $67 \%$ and water in $81 \%$. There was also a lower consumption of unhealthy and ultra-processed foods such as hot-dogs in $90 \%$ of households, sausages in $73 \%$, sugary drinks in 79\% and ice cream in 83\%. Conclusions: The COVID-19 pandemic in Chile increased the perception of food insecurity in households with vulnerable conditions. However, the consumption of healthier foods increased.

(Rev Med Chile 2021; 149: 980-988)

Key words: COVID-19; Chile; Feeding Behavior; Food Insecurity.
L a pandemia por el nuevo coronavirus llamado SARS-CoV-2, que comenzó en Wuhan China, ha registrado una rápida y exponencial propagación mundial, alcanzando todos los países y territorios de las Américas. Los datos reportados en julio de 2020, por la Organización Panamericana de la Salud/Organización Mundial de la Salud (OPS/OMS), comunicaron un total de 7.702.075 casos confirmados y 311.569.248 muertes ${ }^{1}$.

En Chile, el Ministerio de Salud (MINSAL) confirma el primer caso de coronavirus (COVID-19) los primeros días de marzo de 2020, y al 24 de julio se había registrado un total de 341.304 casos acumulados, con un total de fallecidos de 8.914, cifras que aumentan día a día².
Dicha enfermedad ha traído consigo un cambio radical en las actividades diarias realizadas por la población, incorporando el distanciamiento social y medidas de aislamiento propuestas por los gobiernos, tales como las cuarentenas voluntarias u obligatorias, suspensión de clases y jornadas laborales, toques de queda, controles fronterizos $y$ cordones sanitarios ${ }^{3}$.

Estas restricciones han provocado un cambio en los sistemas alimentarios, tanto en la producción, distribución y comercialización, como también en la economía del transporte, envasado y almacenamiento de alimentos ${ }^{4,5}$. Lo anterior influye directamente en la compra y provisión de alimentos en el hogar, especialmente en los más vulnerables (con presencia de niños o adultos 
mayores), propiciando una mayor inseguridad alimentaria, con disminución en los alimentos disponibles para el consumo, preocupación por no poder alimentarse adecuadamente y una probable baja calidad de la dieta (alimentos con alto contenido calórico, de azúcares, grasas saturadas o sodio), lo que se contrapone con recomendaciones de alimentación y nutrición ante la crisis sanitaria del COVID-196.

La OPS/OMS recalca que el derecho a la salud está estrechamente relacionado con otros derechos humanos, como es el derecho a la alimentación, especialmente en sectores vulnerables de la población como son los niños y adultos mayores $^{7}$, lo que hace imperativo mejorar el direccionamiento de las acciones orientadas a mitigar las externalidades negativas que puedan ocurrir. Este estudio tiene como objetivo analizar la inseguridad y los hábitos alimentarios durante el período de confinamiento en un grupo de la población chilena.

\section{Material y Método}

Estudio trasversal, en una muestra por conveniencia, reclutada por Internet a través de la web de 3 instituciones de Educación Superior, 2 públicas y 1 privada (Universidad del Bío-Bío, Universidad del Desarrollo y Universidad de Santiago de Chile). El criterio de inclusión consideró a personas mayores de 18 años y, como criterios de exclusión, personas con discapacidad visual o que no aceptaran contestar el cuestionario. Completaron la totalidad de las preguntas 2.767 personas.

Cada persona debía dar su consentimiento informado y contestar un cuestionario electrónico en la plataforma Google Forms que perseguía conocer la inseguridad alimentaria y hábitos alimentarios en tiempo de coronavirus en el hogar. Este cuestionario fue previamente validado por expertos en nutrición y salud pública, a través de una revisión exhaustiva del instrumento, no publicada.

Se consultó sobre la información sociodemográfica del encuestado (edad, sexo, lugar de residencia, nivel educacional) así como de su grupo familiar (número de integrantes en la familia, presencia de menores de 18 años o adultos mayores). Se construyeron macrozonas a partir de la localización de las comunas de residencia.
Centro-Norte (Región de Arica y Parinacota hasta Región Metropolitana), Centro-Sur (Región de O’Higgins hasta Región de Nuble) y Sur (Región del Biobío a Región de Magallanes). También se incluyó un cuestionario con preguntas sobre seguridad alimentaria basada en el módulo de Seguridad Alimentaria de la Encuesta Nacional de Caracterización Socioeconómica $(\mathrm{CASEN})^{8}$, en cuanto al acceso a alimentos, ingesta en el hogar y preocupación del grupo familiar por no tener alimento en tiempo de pandemia por coronavirus. Además, se consultó sobre los hábitos alimentarios del grupo familiar, especialmente enfocado a las modificaciones en el consumo de grupos de alimentos (consumo más que antes del confinamiento, menos que antes del confinamiento). Se incluyeron grupos de alimentos tales como: lácteos, cereales, pan, legumbres, frutas, verduras, carnes (vacuno, cerdo, pollo), pescados, legumbres, infusiones, agua y también se incluyeron algunos alimentos ultraprocesados y de alta densidad energética (hamburguesas, hotdog/completos, papas fritas, helados y productos de pastelería). La recogida de la información se realizó durante 8 semanas, desde el 30 de marzo al 18 de mayo de 2020 .

El estudio fue desarrollado siguiendo la Declaración de Helsinki ${ }^{9}$ sobre el trabajo con participante humanos y aprobado por los comités de Ética de las tres universidades. Previo a dar respuesta al cuestionario anónimo, los interesados debían leer un consentimiento informado que indicaba detalles sobre el objetivo de la investigación, la participación voluntaria implicaba su consentimiento y la posibilidad de abandonar la respuesta al cuestionario en cualquier momento. Además, se aclaraba que los datos recogidos no serían individualizados.

\section{Análisis estadístico}

Se calcularon frecuencias absolutas y porcentajes con sus intervalos de confianza inferior y superior (IC 95\%). Para analizar el consumo de alimentos durante el confinamiento por coronavirus en los hogares chilenos, se confeccionó un gráfico de barra agrupada que muestra en términos porcentuales las familias que declaran un mayor consumo (desde 1 a 100) y menor consumo (desde -1 a -100), por total de familias y según la presencia de niños, adolescentes y adultos mayores en el hogar. Se utilizó la prueba de Chi-cuadrado 
y test exacto de Fisher, en el caso de frecuencias esperadas menores a 5 .

También se realizó la estimación de los odds ratios (OR) en forma cruda y ajustada con modelos multivariados de regresión logística para analizar la magnitud de la asociación entre la percepción de inseguridad alimentaria con los antecedentes de la familia y lugar de residencia. Para el análisis estadístico de los datos se utilizó el programa estadístico Stata $13^{10}$. Se consideró estadísticamente significativo con un valor de $\mathrm{p}<0,05$.

\section{Resultados}

La muestra estuvo constituida por 2.767 personas, ambos sexos, edad promedio de $26,6 \pm 11,1$ años y mayormente residente en zonas urbanas $(87,3 \%)$. El 25,0\% tenía nivel de educación universitaria y $75,0 \%$ restante lo componen personas con enseñanza media, técnico o estudiante universitario. En 46,0\% de los hogares habitaban menores de 18 años y en 20,8\% adultos mayores de 65 años. Más de la mitad de los hogares estaba constituido por 3 a 4 integrantes $(53,0 \%)$ (Tabla 1$)$.

Tabla 1. Caracterización de la muestra, datos del encuestado y datos del hogar

\begin{tabular}{|c|c|c|c|c|}
\hline \multirow[t]{2}{*}{ Variables } & \multirow[t]{2}{*}{$\mathbf{n}$} & \multirow[t]{2}{*}{$\%$} & \multicolumn{2}{|c|}{ IC $95 \%$} \\
\hline & & & L.inf & L.sup \\
\hline \multicolumn{5}{|l|}{ Datos del encuestado } \\
\hline \multicolumn{5}{|l|}{ Sexo } \\
\hline Femenino & 1.871 & 67,1 & 65,8 & 69,3 \\
\hline Masculino & 896 & 32,3 & 30,6 & 34,1 \\
\hline \multicolumn{5}{|l|}{ Edad } \\
\hline Menos de 20 & 1.025 & 37,0 & 35,2 & 38,8 \\
\hline $21-30$ & 1.103 & 39,8 & 38,0 & 41,7 \\
\hline $31-40$ & 277 & 10,0 & 8,9 & 11,1 \\
\hline $41-50$ & 196 & 7,0 & 6,1 & 8,1 \\
\hline $51-60$ & 117 & 4,2 & 3,5 & 5,0 \\
\hline 61 y más & 49 & 1,7 & 1,3 & 2,3 \\
\hline \multicolumn{5}{|l|}{ Lugar de residencia } \\
\hline Urbano & 2.417 & 87,3 & 86,0 & 88,5 \\
\hline Rural & 350 & 12,6 & 11,4 & 13,9 \\
\hline \multicolumn{5}{|l|}{ Nivel educacional } \\
\hline Estudiante universitario & 1.565 & 56,4 & 54,5 & 58,2 \\
\hline Enseñanza media & 422 & 15,2 & 13,9 & 16,6 \\
\hline Técnico & 101 & 3,6 & 3,0 & 4,4 \\
\hline Universitaria & 682 & 24,6 & 23,0 & 26,2 \\
\hline \multicolumn{5}{|l|}{ Datos del hogar } \\
\hline \multicolumn{5}{|c|}{ En el hogar viven niños/as y adolescentes } \\
\hline Sí & 1.280 & 46,2 & 44,4 & 48,1 \\
\hline No & 1.487 & 53,7 & 51,8 & 55,5 \\
\hline \multicolumn{5}{|c|}{ En el hogar viven mayores de 65 años } \\
\hline Sí & 578 & 20,8 & 19,4 & 22,4 \\
\hline No & 2.189 & 79,1 & 77,5 & 80,5 \\
\hline \multicolumn{5}{|l|}{ Tamaño grupo familiar } \\
\hline 1 integrante & 117 & 4,2 & 3,5 & 5,0 \\
\hline 2 integrantes & 424 & 15,3 & 14,0 & 16,7 \\
\hline 3-4 integrantes & 1.467 & 53,0 & 51,1 & 54,8 \\
\hline 5 o más integrantes & 759 & 27,4 & 25,7 & 29,1 \\
\hline
\end{tabular}


Tabla 2. Preocupación del grupo familiar por no tener alimento suficiente, durante tiempo de coronavirus (datos del hogar)

\begin{tabular}{|c|c|c|c|c|c|}
\hline \multirow[t]{2}{*}{ Variables } & \multicolumn{2}{|c|}{ No se preocupó } & \multicolumn{2}{|c|}{ Sí se preocupó } & \multirow[t]{2}{*}{$\mathbf{p}$} \\
\hline & $\%$ & $\mathbf{n}$ & $\%$ & $\mathbf{n}$ & \\
\hline \multicolumn{6}{|c|}{ Integrantes en la familia } \\
\hline 1 integrante & 53,8 & 63 & 46,1 & 54 & 0,000 \\
\hline 2 integrantes & 47,6 & 202 & 52,3 & 222 & \\
\hline 3-4 integrantes & 39,6 & 582 & 60,3 & 885 & \\
\hline 5 o más integrantes & 38,2 & 290 & 61,7 & 469 & \\
\hline \multicolumn{6}{|c|}{ Hogar viven menores 18 años } \\
\hline No & 44,5 & 622 & 55,4 & 825 & 0,000 \\
\hline Sí & 37,1 & 475 & 62,8 & 805 & \\
\hline \multicolumn{6}{|c|}{ Hogar viven mayores de 65 años } \\
\hline No & 41,5 & 910 & 58,4 & 1.279 & 0,318 \\
\hline Sí & 39,2 & 227 & 60,7 & 351 & \\
\hline \multicolumn{6}{|l|}{ Lugar de residencia } \\
\hline Rural & 32,5 & 114 & 67,4 & 236 & 0,001 \\
\hline Urbana & 42,3 & 1.023 & 57,6 & 1.394 & \\
\hline \multicolumn{6}{|c|}{ Macrozona de residencia } \\
\hline Centro-Norte & 49,7 & 640 & 50,2 & 647 & \\
\hline Centro-Sur & 32,4 & 241 & 67,5 & 501 & 0,000 \\
\hline Sur & 34,6 & 256 & 65,3 & 482 & \\
\hline
\end{tabular}

En la Tabla 2, se observa que en las familias con más de 3 integrantes y cuando en el hogar viven menores de 18 años, la proporción de preocupación del grupo familiar por no tener alimento suficiente durante tiempo de coronavirus superó a $60,0 \%(p=0,000)$. Las personas que habitaban en zonas rurales presentaron mayor proporción de preocupación por tener suficiente alimento, superando en 10 puntos porcentuales a las personas que residían en zonas urbanas $(p=0,001)$. Las familias que vivían en las macrozonas Centro-Sur y Sur señalaron tener mayor preocupación por tener alimentos disponibles en el hogar, superando en hasta 18 puntos porcentuales a las familias que vivían en la zona Centro Norte (50,0\% vs. $68,0 \%$; $\mathrm{p}=0,000)$.

La percepción de inseguridad alimentaria en familias que tenían más de 3-4 integrantes versus las personas que vivían solas fue 1,7 (IC95\% = $1,17-2,49 ; p=0,006)$ veces mayor. Cuando las familias tenían 5 o más integrantes, esta percepción aumentó a 1,79 $(\mathrm{IC} 95 \%=1,21-2,66 ; \mathrm{p}=0,003)$ veces. Por otro lado, las familias que tenían integrantes menores de 18 años tenían una percepción de inseguridad alimentaria 1,34 (IC95\% $=1,15$ $1,56 ; \mathrm{p}=0,000)$ veces mayor en comparación con las que no tenían menores de 18 años en su grupo familiar. Al observar la inseguridad alimentaria por lugar en que habita la familia, aquellas en la macrozona Centro-Sur y Sur tenían una percepción de inseguridad alimentaria mayor respecto a las de la macrozona Centro-Norte (Centro-Sur OR: 1,$96 ;$ IC95\% $=1,61-2,38 ; \mathrm{p}=0,000$ y Sur OR: 1,$85 ;$ IC95\% $=1,53-2,23 ; \mathrm{p}=0,000)$ (Tabla 3$)$.

Durante el período de confinamiento por la pandemia de coronavirus estudiado, cambió el consumo de alimentos en los hogares. En la Figura $1 \mathrm{~A}$, se observa que, del total de familias, sobre $60 \%$ de ellas aumentó el consumo de agua $(80,7 \%)$, té $(86,0 \%)$, café $(67,0 \%)$, verduras $(67,2 \%)$, huevo $(79,0 \%)$, legumbres $(71,9 \%)$ y pan $(66,1 \%)$. Por otra parte, 8 de cada 10 familias señaló haber dis- 
Tabla 3. Asociación entre inseguridad alimentaria y antecedentes de la familia. (Datos del hogar)

\begin{tabular}{|c|c|c|c|c|}
\hline Antecedentes familia & $\begin{array}{l}\text { OR Crudo } \\
\text { (95\% CI) }\end{array}$ & Valor $\mathbf{p}$ & $\begin{array}{l}\text { OR ajustado* } \\
(95 \% \mathrm{CI})\end{array}$ & Valor $\mathbf{p}$ \\
\hline \multicolumn{5}{|l|}{ Integrantes grupo familiar } \\
\hline 1 integrante & 1,00 & & 1,00 & \\
\hline 2 integrantes & $1,28(0,85-1,93)$ & 0,235 & $1,25(0,83-1,89)$ & 0,273 \\
\hline 3-4 integrantes & $1,77(1,21-2,58)$ & 0,003 & $1,70(1,17-2,49$ & 0,006 \\
\hline 5 o más integrantes & $1,88(1,27-2,79)$ & 0,001 & $1,79(1,21-2,66)$ & 0,003 \\
\hline \multicolumn{5}{|c|}{ Familia con integrante menor de 18 años } \\
\hline No & 1,00 & & 1,00 & \\
\hline Sí & $1,35(1,16-1,58)$ & 0,000 & $1,34(1,15-1,56)$ & 0,000 \\
\hline \multicolumn{5}{|c|}{ Familia con integrante mayor de 65 años } \\
\hline No & 1,00 & & 1,00 & \\
\hline Sí & $1,10(0,91-1,32)$ & 0,318 & $1,10(0,913-1,33)$ & 0,308 \\
\hline \multicolumn{5}{|c|}{ Lugar en que habita la familia (Macrozona) } \\
\hline Centro-Norte & 1,00 & & 1,00 & \\
\hline Centro-Sur & $2,05(1,70-2,48)$ & 0,000 & $1,96(1,61-2,38)$ & 0,000 \\
\hline Sur & $1,86(1,54-2,24)$ & 0,000 & $1,85(1,53-2,23)$ & 0,000 \\
\hline
\end{tabular}

*Ajustado por lugar de residencia (urbano, rural).

minuido el consumo de alimentos no saludables, tales como: hamburguesas $(-85,0 \%)$, helados $(-82,9 \%)$, carne de cerdo $(-88,9 \%)$, mientras que más de 90\% de las familias señaló haber disminuido el consumo de hot-dog/completos $(-90,2 \%)$ y mariscos (-92,3\%).

En hogares con menores de 18 años (Figura 1B) aumentó significativamente el consumo de mantequilla $(50,3 \%)$, azúcar $(50,6 \%)$ y jugos azucarados $(51,8 \%)$ y disminuyó el consumo de legumbres $(-51,5 \%)$, mariscos $(-49,8 \%)$, huevos $(-52,8 \%)$, frutas $(-47,4 \%)$, frutos secos $(-51,0 \%)$, verduras $(-47,3 \%)$, mate $(-51,0 \%)$ y hamburguesas $(-48,8 \%)$.

En los hogares con presencia de adultos mayores (Figura 1C), solo se observó una disminución significativa en los productos de pastelería $(-23,1 \%)$.

\section{Discusión}

Los resultados de la presente investigación realizada durante el período de confinamiento por la pandemia COVID-19 muestran que las familias aumentan su percepción de inseguridad alimentaria, especialmente cuando el hogar está compuesto por más de 3 integrantes, en el que viven menores de 18 años que habitan sectores rurales de las zonas del centro y sur del país, las que se encuentran más alejadas de la capital. Es interesante observar que una proporción importante de los hogares ha cambiado sus patrones de consumo, aumentando la ingesta de alimentos saludables (leche, huevos, fruta, verduras, agua). Por otra parte, disminuye el consumo de alimentos no saludables (hot-dog/ completos, embutidos, bebidas azucaradas). Resultados semejantes fueron descritos en España, donde los autores señalan una fuerte tendencia hacia el aumento de consumo de alimentos saludables, disminución del consumo de alimentos no saludables y aumento de la práctica de cocinar en casa $^{11,12}$. Este aumento en la ingesta de alimentos más sanos puede explicarse por el conocimiento de la población sobre la importancia de una alimentación saludable y su vinculación con un mejor estado de salud general, además de la mayor presencia de los integrantes del hogar en el domicilio, lo que puede explicar que las familias extremen sus cuidados alimentarios para no enfermarse. 


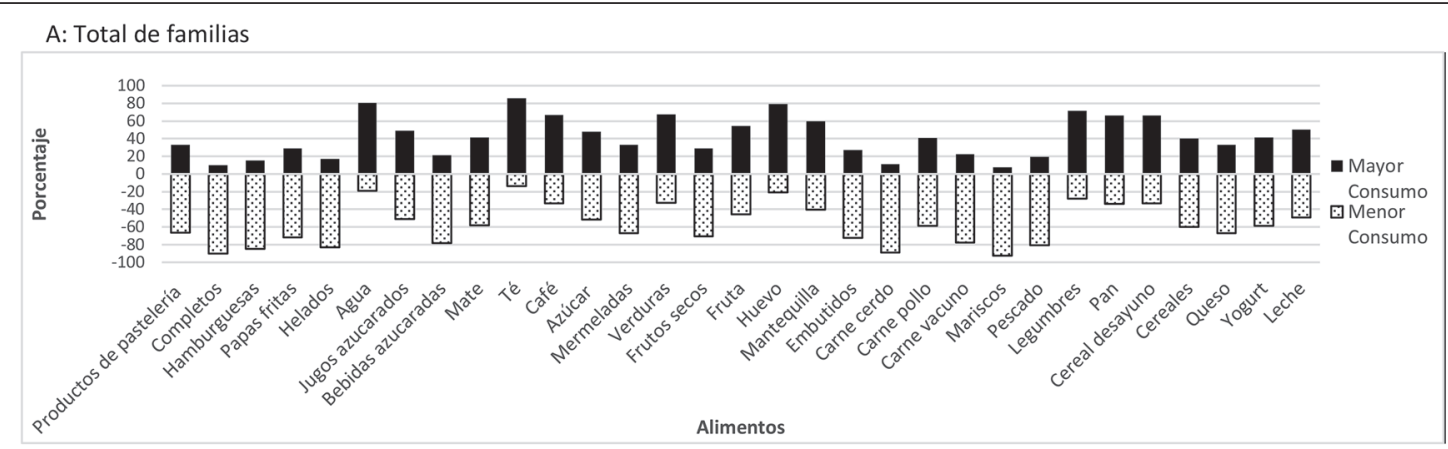

B: Familias con niños y adolescentes

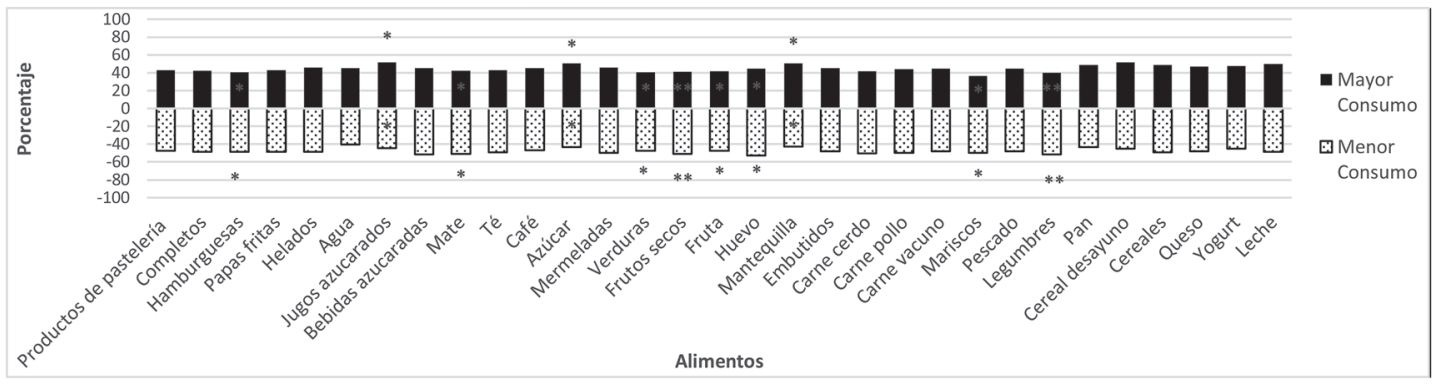

C: Familias con adultos mayores

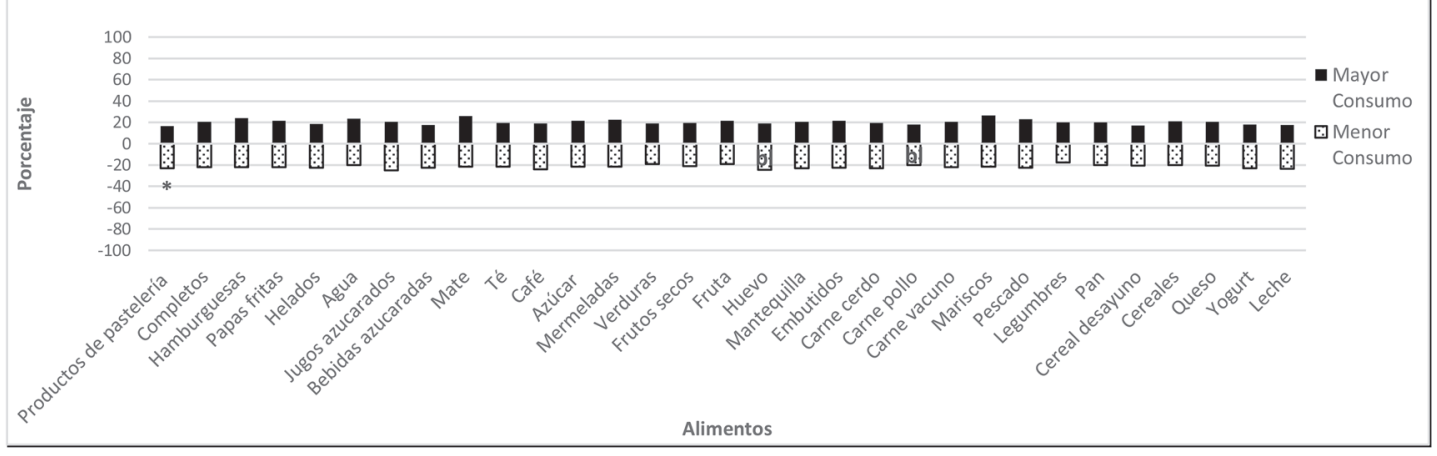

Figura 1. Consumo de alimentos, durante el confinamiento por coronavirus, en el total de hogares, hogares con presencia de adultos mayores y hogares con presencia de menores de 18 años. ${ }^{*} p<0,05 ;{ }^{* *} p>0,01$.

Organizaciones internacionales han reforzado las recomendaciones de mantener una alimentación saludable en tiempo de coronavirus ${ }^{13,14}$, las que están en concordancia con las guías alimentarias (GABAs) para la población chilena ${ }^{15}$.

Contrariamente con lo anterior, otros estudios han demostrado alteración en los patrones de consumo, con aumento tanto en la cantidad como en el tipo de alimentos no saludables consumidos, así como el incremento en la ingesta de snacks entre las principales comidas ${ }^{16}$. Además, se reporta una disminución en el consumo de frutas, vegetales, legumbres y un aumento en el consumo de carnes, lácteos y alimentos de comida rápida ${ }^{17}$.

Respecto a inseguridad alimentaria, especial importancia cobran aquellos hogares con presencia de menores de 18 años que, juntamente con los adultos mayores, son considerados grupos vulnerables en la sociedad. En nuestro estudio, aquellos hogares con presencia de estos grupos evidencian una mayor preocupación por no tener alimentos en cantidad suficiente para cubrir sus 
necesidades. La posible inestabilidad en la empleabilidad refuerza el estado vulnerable de los hogares, requiriendo una mayor protección social, considerando la estrecha relación existente entre alimentación-nutrición, salud y situación socioeconómica. El cambio en el consumo de alimentos en hogares con menores de 18 años, tales como el incremento de azúcar y jugos azucarados y la disminución de otros más saludables, como las frutas, expresa nítidamente los efectos negativos de la pandemia en la vida de las comunidades. Un reciente trabajo que analiza la situación de estos grupos de riesgo indica a la necesidad de una mayor coordinación intersectorial entre los sistemas alimentarios, además de la integración con los sistemas de salud y protección social ${ }^{18}$.

Wolfson y cols. llaman la atención para un aumento en la inseguridad alimentaria en Estados Unidos de Norteamérica, donde menos de 20,0\% de los adultos que presentan muy baja seguridad alimentaria tiene condiciones para cumplir con las recomendaciones de adquisición de alimentos al menos para 15 días. Los autores reafirman que los efectos a corto plazo de la pandemia exacerban las desigualdades existentes, afectando aquellos hogares con inseguridad alimentaria, de bajos ingresos económicos y que no logran cubrir sus necesidades básicas ${ }^{19}$.

En Chile, para aminorar el impacto de la pandemia en la situación de inseguridad alimentaria de los grupos más vulnerables, además de los programas estatales de entrega de alimentos para grupos específicos como los programas alimentarios del Ministerio de Salud ${ }^{20}$, se ha reforzado el Programa de Alimentación Escolar (PAE) ${ }^{21}$, cambiando su modalidad de entrega de beneficio, pasando de la entrega de raciones preparadas a la entrega de cajas de alimentos orientadas a cubrir los requerimientos nutricionales de los escolares por un período de 15 días $^{22}$. Además, el gobierno, a través de las municipalidades, inició la entrega de un beneficio (caja de alimentos) para las familias más vulnerables, el que cubre los requerimientos de calorías y macronutrientes de una familia con 5 integrantes por aproximadamente 10 días $^{23}$.

Nuestros resultados reflejan la urgencia de reforzar la importancia de la alimentación en los planes de emergencia en este período de COVID-19, y particularmente una alimentación saludable, lo que necesariamente requiere considerar alimentos in natura en los programas de asistencia nutricional. Una de las alternativas, y considerando las dificultades logísticas involucradas, puede incluir el potenciamiento de los canales de distribución de alimentos, tales como ferias libres y locales de ventas de frutas y verduras en las comunidades. En estos espacios existen todos los alimentos considerados como imprescindibles, por lo que es relevante brindar mayor seguridad sanitaria para evitar que sean focos de trasmisión de la enfermedad.

A mediano plazo, se hace imprescindible reflexionar sobre medidas fiscales necesarias para disminuir el consumo de alimentos altos en nutrientes críticos, sobre todo si asumimos que el aumento de riesgo de mortalidad por COVID-19, ocurre principalmente en aquellos sujetos que padecen enfermedades vinculadas a la mala alimentación, como la obesidad ${ }^{19}$. Sobre campañas educativas en períodos de pandemias, estas deberían entregar información clara de cómo adquirir, preparar y consumir alimentos en forma segura, priorizando los mensajes de las $\mathrm{GABAs}^{15}$.

La pandemia por COVID-19, en el caso de Chile, más que instaurar una situación de inseguridad alimentaria en grupos específicos de la población, muestra una realidad que ya había sido descrita en la última CASEN, donde más de 25,0\% de la población refiere no tener dinero suficiente para comprar alimentos saludables, llegando a 40,0\% en hogares del primer quintil de ingreso ${ }^{8}$.

Es imperativo repensar el sistema alimentario chileno, donde la priorización en la producción de alimentos más saludables está orientada al mercado externo. Productos alimentarios por los cuales Chile es reconocido mundialmente tienen muy bajo consumo interno, como es el caso de los pescados, frutas y verduras, lo que viene siendo evidenciado en las últimas encuestas nacionales que evalúan consumo ${ }^{24-27}$.

Reposicionar y promover medidas fiscales necesarias para disminuir el consumo de alimentos altos en nutrientes críticos, tales como impuestos saludables y aumentar el consumo de alimentos saludables, requiere, sobre todo en Chile, reconocer que la inseguridad alimentaria no está necesariamente vinculada a la escasez de alimentos. Esta se relaciona más a condiciones de vulnerabilidad como pobreza e inequidades sociales, problemas en la distribución geográfica de los canales de distribución de alimentos saludables, además de una incipiente política de Estado orientada 
a proveer el mercado interno con alimentos en calidad y precios accesibles a todos los grupos de la población. Soberanía y seguridad alimentaria deben ser conceptos clave en los lineamientos políticos en distintas áreas y debe ser uno de los principales enunciados en la Constitución del país, con miras a la instalación de ambientes alimentarios más saludables, donde la academia juega un rol importante ${ }^{28-31}$.

Una de las debilidades de nuestro estudio se refiere a las limitaciones propias del diseño, ya que se trata de un estudio transversal con una muestra por conveniencia y, por lo tanto, solo muestra la realidad de los hogares en el inicio de la pandemia. Además, también por limitaciones del diseño (encuesta on line) no fue posible llegar a aquellos hogares más vulnerables y que ciertamente fueron los más afectados por la situación de pandemia.

\section{Referencias}

1. PAHO. Informes de situación de la COVID-19, OPS/ OMS, Organización Panamericana de la Salud [consultado el 03 de agosto de 2020]. Disponible: https://www. paho.org/es/tag/informes-situacion-covid-19

2. Ministerio de Salud. COVID-19 en Chile: La realidad nacional en datos [consultado el 31 de julio de 2020]. Disponible: https://www.gob.cl/coronavirus/cifrasoficiales/

3. Nicola M, Alsafi Z, Sohrabi C, Kerwan A, Al-Jabir A, Iosifidis $\mathrm{C}$, et al. The socio-economic implications of the coronavirus pandemic (COVID-19): A review. Int J Surg 2020; 78: 185-93.

4. Shanks CB, Hingle MD, Parks CA, Yaroch AL. The COVID-19 Pandemic: A Watershed Moment to Strengthen Food Security Across the US Food System. Am J Public Health 2020, 110: 1133-4.

5. Oliveira TC, Abranches MV, Lana RM. (In) Segurança alimentar no contexto da pandemia por SARS-CoV-2. Cad Saude Publica. 2020; 36(4):e00055220.

6. Academia Española de Nutrición y Dietética, Consejo General de Colegios Oficiales de Dietistas-Nutricionistas. Recomendaciones de alimentación y nutrición para la población española ante la crisis sanitaria del COVID-19 [consultado el 31 de julio de 2020].Disponible: https://academianutricionydietetica.org/NOTICIAS/ alimentacioncoronavirus.pdf

7. PAHO. Promoción de la equidad en la salud, la igualdad étnica y de género, y los derechos humanos en la respuesta a la COVID-19: consideraciones clave [con- sultado el 30 de julio de 2020]. Disponible: https://iris. paho.org/bitstream/handle/10665.2/52075/OPSEGCLEGCOVID190001_spa.pdf?sequence=1\&isAllowed =y

8. Ministerio de Desarrollo Social. Encuesta de Caracterización Sioeconómica (CASEN)- Inseguridad Alimentaria Síntesis de Resultados [consultado el 31 de julio de 2020]. Disponible: http://observatorio.ministeriodesarrollosocial.gob.cl/casen-multidimensional/casen/docs/ CASEN_2017_Inseguridad_alimentaria.pdf

9. Asamblea Médica Mundial. Declaración de Helsinki de la AMM - Principios éticos para las investigaciones médicas en seres humanos [consultado el 15 de marzo de 2020]. Disponible: https://medicina.udd.cl/centro-bioetica/files/2015/10/Declaracion-Helsinski-2013.pdf

10. Stata Corp. Stata Statistical Software: Release 13.0 College Station, TX: StataCorp LP.

11. Pérez-Rodrigo C, Citores MG, Hervás Bárbara G, Litago FR, Casis Sáenz L, Aranceta-Bartrina J, et al. Cambios en los hábitos alimentarios durante el período de confinamiento por la pandemia COVID-19 en España. Rev Esp Nutr Comunitaria 2020; 26 (2): 28010.

12. Rodríguez-Pérez C, Molina-Montes E, Verardo V, Artacho R, García-Villanova B, Guerra-Hernández EJ, et al. Changes in dietary behaviours during the COVID-19 outbreak confinement in the Spanish COVIDiet study. Nutrients 2020; 12 (6): 1-19.

13. Wold Health Organization. Food and nutrition tips during self-quarantine [consultado el 15 de julio de 2020]. Disponible: https://www.euro.who.int/en/health-topics/ health-emergencies/coronavirus-covid-19/technical-guidance/food-and-nutrition-tips-during-self-quarantine

14. United States Department of Agriculture. Start Simple with MyPlate: Food Planning During the Coronavirus Pandemic. ChooseMyPlate [consultado el 3 de agosto de 2020]. Disponible: https://www.choosemyplate.gov/ coronavirus

15. Ministerio de Salud. Informe final "Estudio para revisión y actualziación de las Guías Alimentarias para la Población Chilena " [consultado el 13 de abril de 2020]. Disponible: http://www.minsal.cl/portal/url/item/ dde0bc471a56a001e040010165012224.pdf

16. Ammar A, Brach M, Trabelsi K, Chtourou H, Boukhris O, Masmoudi L, et al. Effects of COVID-19 Home Confinement on Eating Behaviour and Physical Activity: Results of the ECLB-COVID19 International Online Survey. Nutrients 2020; 12 (6): 1583.

17. Sidor A, Rzymski P. Dietary Choices and Habits during COVID-19 Lockdown: Experience from Poland. Nutrients 2020; 12 (6): 1657.

18. Pérez Escamilla R, Cunningham K, Moran VH. COVID-19 and maternal and child food and nutrition 
insecurity: a complex syndemic. Matern Child Nutr 2020; 16 (3): e13036.

19. Wolfson JA, Leung CW. Food Insecurity and COVID-19: Disparities in Early Effects for US Adults. Nutrients 2020; 12 (6): 1648.

20. Ministerio de Salud. Norma Técnica de los Programas Alimentarios. [Consultado el 01 de mayo de 2020]. Disponible: http://web.minsal.cl/wp-content/ uploads/2015/09/2016.04.20-Norma-Técnica-Prog.-Alimentarios-aprobada-por-Jurídica.pdf

21. Junta Nacional de Auxilio Escolar y Becas. Programa de Alimentación Escolar (PAE) [consultado el 20 de julio de 2020]. Disponible: https://www.junaeb.cl/programa-de-alimentacion-escolar

22. Ministerio de Educación. Suspensión de clases: Se repartirá canastas de alimentación a estudiantes [consultado el 3 de agosto de 2020]. Disponible: https://www.mineduc.cl/suspension-de-clases-mineduc-repartira-canastas-de-alimentacion-a-estudiantes/

23. Ministerio de Desarrollo Social y Familia. Gobierno anuncia nueva entrega de Alimentos para Chile que beneficiará a 3 millones de familias [consultado el 3 de agosto de 2020]. Disponible: http://www.desarrollosocialyfamilia.gob.cl/noticias/gobierno-anuncia-nueva-entrega-de-alimentos-para-chile-que-beneficiara-a-3-millones-de-familias

24. Ministerio de Salud. Encuesta Nacional de Salud 20162017 [consultado 15 de mayo de 2020]. Disponible: http://epi.minsal.cl/ens-resultados-ens-2016-2017/

25. Ministerio de Salud. Encuesta Nacional de Consumo
Alimentario. Informe Final. [consultado el 15 de mayo de 2020]. Disponible: http://web.minsal.cl/sites/default/ files/ENCA-INFORME_FINAL.pdf

26. Araneda J, Pinheiro A, Rodríguez L, Rodríguez A. Consumo aparente de frutas, hortalizas y alimentos ultraprocesados en la población chilena. Rev Chil Nutr 2016; 43 (3): 271-8.

27. Pinheiro A, Ivanovic C, Rodríguez L. Consumo de legumbres en Chile. Perspectivas y desafíos. Rev Chil Nutr. 2018; 45 (4): 14-20.

28. Zamora P, Rodríguez L, Pinheiro A, Pizarro T. Política Nacional de Alimentación y Nutrición [consultado el 15 de mayo de 2020]. Disponible: http://www.bibliotecaminsal.cl/wp/wp-content/uploads/2018/01/BVS_POLÍTICA-DE-ALIMENTACIÓN-Y-NUTRICIÓN.pdf

29. Rioseco R, Egaña D, Galvez P, Masferrer D. Marco conceptual sobre los factores condicionantes de los ambientes alimentarios en Chile. Ministerio de Salud. [consultado el 16 de mayo de 2020]. Disponible: http://www.bibliotecaminsal.cl/marco-conceptual-sobre-los-factores-condicionantes-de-los-ambientes-alimentarios-en-chile/

30. Araneda J, Pinheiro A, Rodríguez L. Una mirada actualizada sobre los ambientes alimentarios y obesidad. Rev Chil Salud Pública. 2020; 24 (1): 67-71.

31. Pinheiro A, Araneda J, García Y, Popelka R, González A, Martínez R, et al. Food environments for a healthy and nutritious diet: The contribution of academia. In: Food environments: Where people meet the food system. UNSCN Nutr. 2019; 44: 160-8. 